\title{
Attenuation of Listeria monocytogenes Virulence by Cannabis sativa L. Essential Oil
}

\author{
Emanuela Marini ${ }^{1}$, Gloria Magi ${ }^{1}$, Gianna Ferretti ${ }^{2}$, Tiziana Bacchetti ${ }^{3}$, Angelica Giuliani ${ }^{4}$, \\ Armanda Pugnaloni ${ }^{4}$, Maria Rita Rippo ${ }^{4}$ and Bruna Facinelli ${ }^{1 *}$ \\ ${ }^{1}$ Unit of Microbiology, Department of Biomedical Sciences and Public Health, Polytechnic University of Marche, Ancona, \\ Italy, ${ }^{2}$ Department of Clinical Sciences, Polytechnic University of Marche, Ancona, Italy, ${ }^{3}$ Department of Life and \\ Environmental Sciences, Polytechnic University of Marche, Ancona, Italy, ${ }^{4}$ Division of Pathology, Department of Clinical and \\ Molecular Sciences, Polytechnic University of Marche, Ancona, Italy
}

OPEN ACCESS

Edited by:

You-Hee Cho,

CHA University, South Korea

Reviewed by:

Arun K. Bhunia,

Purdue University, United States

Tânia Aparecida Tardelli Gomes, Federal University of São Paulo, Brazil

*Correspondence:

Bruna Facinelli

b.facinelli@univpm.it

Received: 17 May 2018

Accepted: 31 July 2018

Published: 22 August 2018

Citation:

Marini E, Magi G, Ferretti G

Bacchetti T, Giuliani A, Pugnaloni A,

Rippo MR and Facinelli B (2018)

Attenuation of Listeria monocytogenes Virulence by Cannabis sativa $L$.

Essential Oil.

Front. Cell. Infect. Microbiol. 8:293.

doi: 10.3389/fcimb.2018.00293
Anti-virulence strategies are being explored as a novel approach to combat pathogens. Such strategies include inhibition of surface adhesion, tissue invasion, toxin production, and/or interference with the gene regulation of other virulence traits. Listeria monocytogenes, the causative agent of listeriosis, is a facultative intracellular food pathogen characterized by a wide distribution in the environment. Its ability to persist within biofilms and to develop resistance to sanitizers is the cause of significant problems in food processing plants and of steep costs for the food industry. In humans, the treatment of listeriosis is hampered by the intracellular location of listeriae and the poor intracellular penetration of some antibiotics. Eleven L. monocytogenes isolates from patients who were diagnosed with invasive listeriosis in Italy in 2014-2016 were studied. This in vitro and in vivo study explored the antibacterial and anti-virulence properties of a steam-distilled essential oil of Cannabis sativa L., which is being intensively investigated for its high content in powerful bioactive phytochemicals. Susceptibility experiments demonstrated a moderate bactericidal activity of the essential oil (Minimum Bactericidal Concentration $>2048 \mu \mathrm{g} / \mathrm{mL}$ ). Assessment of the effects of sublethal concentrations of the essential oil on $L$. monocytogenes virulence traits demonstrated a significant action on motility. Listeriae were non-motile after exposure to the essential oil. Light and scanning electron microscopy documented aggregates of listeriae with the flagella trapped inside the cluster. Real-time RT-PCR experiments showed downregulation of flagellar motility genes and of the regulatory gene prfA. The ability to form biofilm and to invade Caco-2 cells was also significantly reduced. Galleria mellonella larvae infected with $L$. monocytogenes grown in presence of sublethal concentrations of the essential oil showed much higher survival rates compared with controls, suggesting that the extract inhibited tissue invasion. Food contamination with L. monocytogenes is a major concern for the food industry, particularly for plants making ready-to-eat and processed food. The present work provides a baseline in the study of the anti-virulence properties of the $C$. sativa essential oil against $L$. monocytogenes. Further studies are needed to understand if it could be used as an alternative agent for the control of $L$. monocytogenes in food processing plants.

Keywords: Cannabis sativa, essential oil, sublethal concentrations, Listeria monocytogenes, virulence, motility, cell invasion, biofilm 


\section{INTRODUCTION}

In recent years, the use of plant products as alternative/adjunct antimicrobial agents to control pathogenic microorganisms has been attracting mounting interest. Anti-virulence strategies are being explored as a novel approach to combat bacterial pathogens; such approaches include inhibition of surface adhesion, tissue invasion, toxin production, and/or interference with the gene regulation of other virulence traits (Rasko and Sperandio, 2010). A major group of plant antimicrobial compounds is represented by essential oils (EO), complex mixtures of volatile secondary metabolites belonging to different chemical families including terpenes, alcohols, ethers, aldehydes, and phenols (Cannas, 2016). The antimicrobial activity of EO and their components has been known for millennia. More recently, synergy between EO components and antibiotics has been demonstrated against antibiotic-resistant pathogens (Langeveld et al., 2014). In contrast, the knowledge of the effects of EO on bacterial virulence is still very limited (Nazzaro et al., 2013; Silva et al., 2016).

Cannabis sativa L. (Cannabaceae), an annual species that has spread from its native range in central Asia to Europe and Africa, is one of the earliest domestic plants in the history of mankind and has been grown and selected for thousands of years for a multiplicity of purposes, like a source of food, fuel, paper, and building material, as a textile fiber, and as a remedy in folk medicine (Andre et al., 2016). The plant is a source of several bioactive compounds including psychoactive substances such as cannabinoids (the most important being $\Delta 9$-tetrahydrocannabinol, $\Delta 9$-THC), terpenoids, flavonoids, and polyunsaturated fatty acids. In the late 1930s, the psychotropic effects due to $\triangle 9$-THC led to a ban on its cultivation worldwide. In recent years, selection of some genotypes containing low $\Delta 9$ THC concentrations $(\leq 0.2 \% \mathrm{w} / \mathrm{v})$ has led to a lifting of the ban; the plant can now be grown legally (Holler et al., 2008) and used for research purposes.

Listeria monocytogenes, a Gram-positive, facultative intracellular pathogen with a wide environmental distribution, is the causative agent of human and animal listeriosis (Freitag et al., 2009). The disease is the most lethal zoonosis in the EU (fatality rate, $20 \%$ ), affecting particularly immunocompromised and elderly individuals (EFSA and ECDC, 2016). Infection of the human host is commonly via the oral route, through ingestion of contaminated food, although transplacental transmission during gestation also occurs. Severe L. monocytogenes infection include septicaemia, meningitis, endocarditis, and spontaneous abortion (McDougal and Sauer, 2018). Infection requires bacterial internalization into host cells, intracellular survival, and spread into neighboring cells, which enable bacterial diffusion from the primary site of infection, usually the bowel, to the liver, the spleen, and on to peripheral blood and eventually the brain. A limited subset of serotypes (i.e., $1 / 2 \mathrm{a}, 1 / 2 \mathrm{~b}$, and $4 \mathrm{~b}$ ) is responsible for the bulk of clinical cases worldwide (Kathariou, 2002); the serotype most frequently associated with listeriosis outbreaks, particularly in Europe and North America, is 1/2a (Lomonaco et al., 2015). L. monocytogenes is intrinsically resistant to broadspectrum cephalosporin antibiotics (Krawczyk-Balska and
Markiewicz, 2016) and shows acquired resistance to other drugs. Treatment with aminopenicillin or benzylpenicillin, alone or combined with an aminoglycoside, is currently the gold standard antibiotic therapy for L. monocytogenes infections (Thønnings et al., 2016). However, listeriae are difficult to eradicate, due to their intracellular location and the poor intracellular penetration of these drugs. Their ability to persist within biofilms and to develop resistance to sanitizers is the cause of significant problems in food processing plants and of steep costs for the food industry (Colagiorgi et al., 2017; Boqvist et al., 2018).

The virulence of $L$. monocytogenes relies on highly effective mechanisms of invasion and spread, which ensure its intracellular lifecycle (Vázquez-Boland et al., 2001; Freitag et al., 2009; Radoshevich and Cossart, 2018). Host cell invasion is either via phagocytosis or receptor-mediated endocytosis, facilitated by the bacterial surface proteins internalins InlA (inlA) and InlB (inlB) (Chen et al., 2017). Recently, Drolia et al. have demonstrated that Listeria adhesion protein (LAP) induces intestinal epithelial barrier dysfunction contributing to bacterial translocation from the intestinal lumen, across the gut epithelium (Drolia et al., 2018). L. monocytogenes is initially trapped in a phagocytic vacuole and subsequently secretes the cholesteroldependent pore-forming toxin listeriolysin O (LLO; $h l y)$ and two phospholipases $\mathrm{C}$ ( $\mathrm{PlcA}$ and $\mathrm{PlcB}$ ), resulting in vacuole rupture and escape of bacteria into cytosol (Chen et al., 2017). In this phase, an actin assembly-inducing protein (ActA; actA) promotes actin-based motility and cell-to-cell spread (Chen et al., 2017). These processes are closely regulated, mainly by the transcriptional regulator PrfA ( $\operatorname{prfA}$ ), which is activated by host infection (de las Heras et al., 2011). Recent progress highlights that $L$. monocytogenes, which has long been a model for cytosolic pathogens, is also capable of residing in vacuoles, in a slow/nongrowing state (Bierne et al., 2018).

Flagellum-based motility and biofilm formation have also been implicated in virulence (Josenhans and Suerbaum, 2002; Duan et al., 2013). The biosynthesis of flagella is temperaturedependent, since most $L$. monocytogenes strains produce flagella and are motile only at temperatures of $30^{\circ} \mathrm{C}$ and less (Peel et al., 1988). Flagellum-mediated motility is critical both for initial surface attachment and subsequent biofilm formation (Lemon et al., 2007).

The purpose of this study was to investigate the in vitro and in vivo antibacterial and anti-virulence properties of an EO extracted from a legal C. sativa L. variety against $L$. monocytogenes isolates collected from patients diagnosed with invasive listeriosis in central Italy in 2014-2016.

\section{MATERIALS AND METHODS}

\section{Strains and Growth Media}

A total number of $11 \mathrm{~L}$. monocytogenes isolates collected from the blood or cerebrospinal fluid (CSF) of patients diagnosed with invasive listeriosis in Ancona province (Italy) from 2014 to 2016 were investigated in the study (Table 1). The 2015 and 2016 isolates had already been characterized in terms of antibiotic resistance phenotype and molecular typing/genetic relatedness (Marini et al., 2016). 
Blood agar base (BAB) supplemented with 5\% sheep blood, Müller-Hinton agar (MHA) supplemented with 5\% sheep blood, Müller-Hinton cation-adjusted broth (CAMHB) supplemented with $3 \%$ laked sheep blood, brain heart infusion (BHI) agar and broth, Tryptone Soya Broth (TSB), and Luria Bertani (LB) agar and broth (all from Oxoid, Basingstoke, UK) were used in the study. Isolates were maintained in glycerol at $-70^{\circ} \mathrm{C}$ and subcultured twice on BAB before testing.

\section{Extraction and Characterization of $\boldsymbol{C}$. sativa L. Essential Oil}

EO was extracted from the French monoecious variety Futura 75, which is grown in the countryside around Ancona. Fresh inflorescences and leaves were steam-distilled by the Associazione Produttori Piante Officinali (APPO Marche, Ancona, Italy). Gas chromatography/mass spectrometry (GCMS) identification of EO components was performed by Agenzia Servizi Settore Agroalimentare delle Marche (ASSAM, Ancona, Italy).

\section{Susceptibility Tests}

The minimum inhibitory concentration (MIC), i.e., the lowest EO concentration inhibiting visible bacterial growth after incubation, was determined in blood-supplemented CAMHB by the microdilution method according to Clinical and Laboratory Standards Institute (CLSI) guidelines (Clinical Laboratory Standards Institute, 2017). The minimum bactericidal concentration (MBC), i.e., the lowest EO concentration killing 99.9\% of the inoculum, was determined by plating $10 \mu \mathrm{L}$ of each microdilution on blood-supplemented MHA followed by overnight incubation at $37^{\circ} \mathrm{C}$. All experiments were performed in triplicate.

\section{Motility Assays}

Motility of L. monocytogenes in presence of the C. sativa EO was assessed by the "umbrella test" in semi-solid agar stabbed with the strains and incubated overnight at $30^{\circ} \mathrm{C}$. Detection of an umbrella-like growth about $0.5 \mathrm{~cm}$ below the agar surface indicated a positive test. Motility was also assessed by measuring the diameter of the growth zone with respect to control bacteria on $0.3 \%$ soft agar plates inoculated with $10 \mu \mathrm{L}$ of a broth culture and incubated overnight at $30^{\circ} \mathrm{C}$.

\section{Flagella Stain}

L. monocytogenes incubated at $30^{\circ} \mathrm{C}$ in presence of the C. sativa EO were examined by light microscopy (LM). Flagella were visualized using BD Flagella Stain Droppers (Becton Dickinson, Milano, Italy), a procedure that demonstrates bacterial flagella and their arrangement on the cell.

\section{Scanning Electron Microscopy Examination}

For morphological studies, L. monocytogenes cells grown on BAB agar at $30^{\circ} \mathrm{C}$ with and without the $C$. sativa $\mathrm{EO}$ were fixed overnight in $2.5 \%$ glutaraldehyde in $0.1 \mathrm{M}$ sodium cacodylate buffer ( $\mathrm{pH} 7.4)$ at $4{ }^{\circ} \mathrm{C}$ and washed with the same buffer supplemented with $7 \%$ sucrose. Samples were left to adhere on glass slides covered with $0.01 \%$ poly-L-lysine solution (Sigma

TABLE 2 | List of primers used in the study.

\begin{tabular}{lllc}
\hline Gene & Primer & Sequence $\left(\mathbf{5}^{\prime} \mathbf{-} \mathbf{3}^{\prime} \mathbf{)}\right.$ & Product size \\
\hline $16 S$ & rDNA 16s-F & AGGTGGGGATGACGTCAAAT & $138 \mathrm{bp}$ \\
& rDNA 16s-R & GCAGCCTACAATCCGAACTG & \\
prfA & prfA-RT-F & GACCGCAAATAGAGCCAAGC & $122 \mathrm{bp}$ \\
& prfA-RT-R & ACTGAGCAAGAATCTACGCAC & \\
flaA & flaA-RT-F & GGCTGCTGAAATGTCCGAAA & \multirow{2}{*}{$90 \mathrm{bp}$} \\
& flaA-RT-R & ATTGCGGTGTTGGTTG & \\
motA & motA-RT-F & GGTACGTACTTGGACGCC & $137 \mathrm{bp}$ \\
& motA-RT-R & AAACGTTCTCCACAACCCG & \\
motB & motB-RT-F & CGTCTGTTGCCTCCAGT & $103 \mathrm{bp}$ \\
& motB-RT-R & ATATGCTTGATTGCCTGCCG &
\end{tabular}

TABLE 1 | L. monocytogenes strains used in the study and their characteristics.

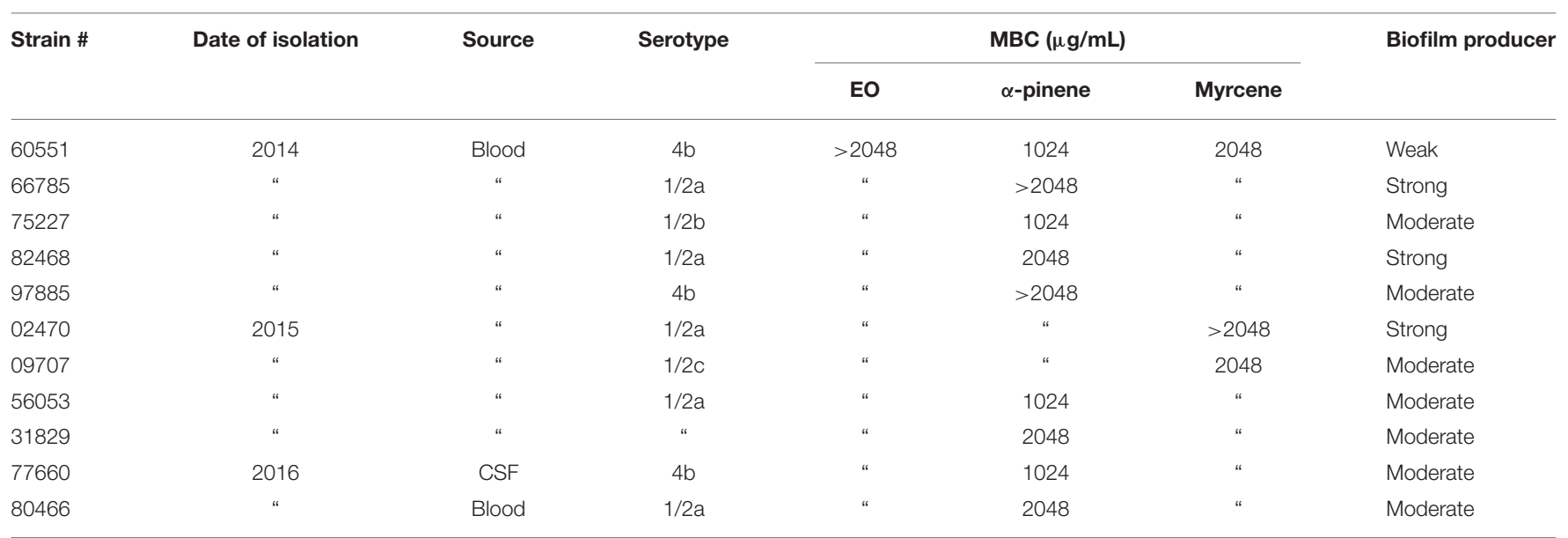

CSF, cerebrospinal fluid; MBC, minimum bactericidal concentration. 
Aldrich, Milano, Italy), postfixed in $1 \% \mathrm{OsO}_{4}$ in $0.1 \mathrm{M}$ sodium cacodylate buffer for $2 \mathrm{~h}$ at $4^{\circ} \mathrm{C}$, washed with the same buffer, dehydrated with $\mathrm{EtOH}$, and critical point dried. Samples were mounted on aluminum stubs by graphite glue (Sigma Aldrich) and coated with a thin $(20 \AA)$ gold film using an EMITECH K550 (Ashford, England) sputtering device. Scanning electron microscopy (SEM) observations were performed with a Zeiss Supra 40 apparatus.

\section{RNA Extraction and Real-Time Quantitative Reverse Transcription PCR (RT-qPCR) on prfA, flaA, motA, and motB}

The effect of the EO on the expression of L. monocytogenes virulence genes was investigated using real time RT-PCR. Strains were grown in presence of $\mathrm{EO}$ at $30^{\circ} \mathrm{C}$ in $\mathrm{TSB}$, and total RNA was extracted using GenElute total RNA Purification kit (Sigma Aldrich). cDNA synthesis was performed using the Quantitect Reverse Transcription kit (Qiagen, Hilden, Germany) and synthesized cDNA was used as the RT-qPCR template. The amplification product was detected using SYBR Green reagents (SYBR Green JumpStar Taq ReadyMix, Sigma Aldrich) by RotorGene (Qiagen). The primers for each gene are reported in Table 2. Data were normalized to the endogenous control (16S rRNA) and the level of candidate gene expression between treated and untreated samples was compared to study relative gene expression and the effect of $\mathrm{EO}$ on each gene.

\section{Biofilm Formation Assays}

Biofilm formation was evaluated as previously described (Marini et al., 2015). Briefly, bacteria were grown overnight in TSB containing $1 \%$ glucose at $30^{\circ} \mathrm{C}$. Overnight bacterial suspensions were prepared to yield final inocula of $\sim 1 \times 10^{8}$ colony-forming units (CFU)/mL; then $200 \mu \mathrm{L}$ aliquots of the bacterial suspension were inoculated into 96-well microtitre plates at least in triplicate. After 24-h incubation, wells were washed 3 times in phosphatebuffered saline (PBS), dried for $1 \mathrm{~h}$ at $60^{\circ} \mathrm{C}$, and stained with Hucker's crystal violet. After three washes in sterile water, wells were inoculated with $100 \mu \mathrm{L}$ of $95 \% \mathrm{EtOH}$ and shaken for $10 \mathrm{~min}$. Biofilm formation was quantified by measuring absorbance at $690 \mathrm{~nm}$ with a Multiscan Ascent apparatus (Thermo Scientific, Waltham, MA, USA). The optical density (OD) cut-off (ODc) was defined as 3 standard deviations above the mean OD of the negative control, represented by non-inoculated wells containing TSB (Stepanovic et al., 2000). Strains were classified as nonproducers $(\mathrm{OD} \leq \mathrm{ODc})$, weak producers (ODc $<\mathrm{OD} \leq 2 \times$ $\mathrm{ODc})$, moderate producers $(2 \times \mathrm{ODc}<\mathrm{OD} \leq 4 \times \mathrm{ODc})$, or strong producers ( $\mathrm{OD}>4 \times \mathrm{ODc})$. The biofilm-forming strain S. epidermidis ATCC 35984 was used as a positive control (Christensen et al., 1985). In some assays, biofilm formation was evaluated in presence of the C. sativa EO. Briefly, overnight bacterial suspensions were prepared to yield final inocula of $\sim 2$ $\times 10^{8} \mathrm{CFU} / \mathrm{mL}$. Then, $100 \mu \mathrm{L}$ of bacterial culture and $100 \mu \mathrm{L}$ of different EO concentrations were added to each well of a 96-well microplate. Wells containing $100 \mu \mathrm{L}$ of the bacterial suspension and $100 \mu \mathrm{L}$ TSB without EO were the positive controls. After incubation, biofilm formation was evaluated as described above. All experiments were performed in triplicate.

\section{Caco-2 Invasion Assays}

The human colon carcinoma Caco-2 cell line (ATCC HTB37) (Rousset, 1986) was used in cell invasion experiments. Cells were routinely cultured in Modified Eagle Medium (MEM; Gibco, New York, NY, USA) supplemented with 1\% (v/v) L-glutamine, $1 \%(\mathrm{v} / \mathrm{v})$ non-essential amino acids and $10 \%(\mathrm{v} / \mathrm{v})$ fetal calf serum (both from Gibco) in $50 \mathrm{~mL}\left(25 \mathrm{~cm}^{2}\right)$ plastic tissue culture flasks (Corning Costar, Milano, Italy) at $37^{\circ} \mathrm{C}$ in an atmosphere containing $5 \% \mathrm{CO}_{2}$. Monolayers were trypsinized and adjusted to a concentration of $2.5 \times 10^{5}$ cells $/ \mathrm{mL}$ in culture medium; $1 \mathrm{~mL}$

TABLE 3 | GC-MS analysis of the C. sativa EO.

Components

\section{1,8-cineole}

Trans- $\beta$-ocime

$\gamma$-terpinene

Terpinolene

Neo-allo-ocimene

Camphor

Borneol

Terpinen-4-ol

$\alpha$-terpinene

Cuminol

Verbenone

Bornyl acetate

Ethyl hexanoate

Iso-caryophyllene

$\beta$-caryophyllene

Allo-aromadendrene

Trans- $\beta$-farnesene

$\alpha$-humulene

$\gamma$-selinene

$\beta$-selinene

$\alpha$-selinene

Valencene

Caryophyllene-oxide

$\alpha$-bisabolol

Components present at greater than $3 \%$ are indicated by bold font.

0.63

0.09

$\mathbf{1 9 . 2 0}$

0.65

4.34

17.17

0.60

0.15

1.81

0.86

1.06

1.52

2.28

0.12

4.50

0.14

5.78

1.15

0.28

0.46

0.64

0.95

0.56

0.08

0.94

15.31

0.11

1.11

3.68

0.28

1.35

1.08

1.22

3.27

0.61 
cell suspension was dispensed into each $22-\mathrm{mm}$ well of a 12 -well tissue culture plate and incubated 4 days to obtain confluent monolayers (Facinelli et al., 1998).

Invasion experiments were performed using $L$. monocytogenes strains grown in presence of the C. sativa EO. Briefly, after overnight growth in $\mathrm{BHI}$ broth supplemented with $\mathrm{EO}$, listeriae were resuspended in PBS to $\mathrm{OD}_{550} 0.6 \pm 0.02$. Then, $1 \mathrm{~mL}$ of the suspension, suitably diluted in MEM, was added to each well to obtain a multiplicity of infection of about 30 bacteria/cell. Penetration was allowed to occur for $1 \mathrm{~h}$ at $37^{\circ} \mathrm{C}$ in $5 \% \mathrm{CO}_{2}$. Then monolayers were washed 3 times and covered with $2 \mathrm{~mL}$ MEM containing a bactericidal concentration of gentamicin $(10 \mu \mathrm{g} / \mathrm{mL})$, to kill extracellular bacteria. After $2 \mathrm{~h}$ at $37^{\circ} \mathrm{C}$ in $5 \% \mathrm{CO}_{2}$ air, cells were washed 3 times and lysed in $1 \mathrm{~mL}$ Triton X-100 (0.1\% in cold sterile water). The CFU of viable bacteria were counted by plating suitable dilutions of the lysates on BHI agar and incubating them for $36-48 \mathrm{~h}$ at $37^{\circ} \mathrm{C}$. Data are expressed as a percentage of initial

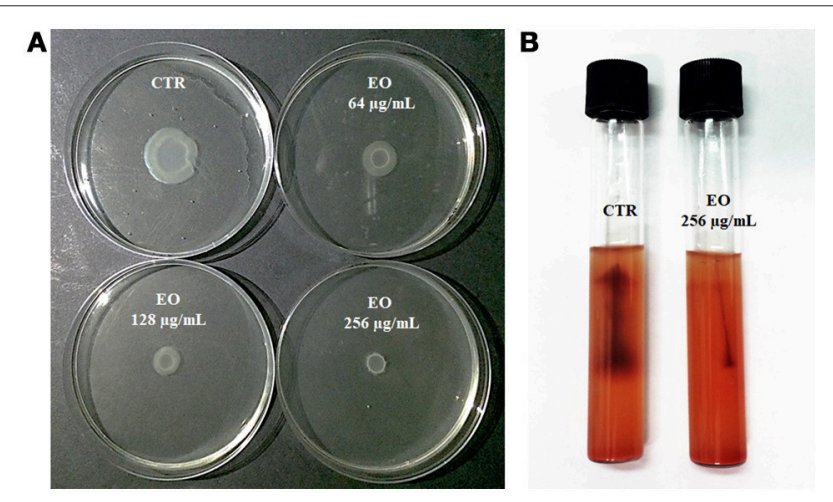

FIGURE 1 | Motility of $L$. monocytogenes strain \#80466 in presence of the $C$. sativa EO. (A) Soft agar motility assay; (B) Umbrella motility test. CTR, control (L. monocytogenes strain grown in the absence of EO).

TABLE 4 | Motility of L. monocytogenes grown in presence of the C. sativa EO

\begin{tabular}{|c|c|c|c|}
\hline \multirow[t]{2}{*}{ Strain \# } & \multicolumn{2}{|c|}{ Zone of motility (mm) } & \multirow[t]{2}{*}{$\%$ reduction } \\
\hline & CTR & EO $(256 \mu \mathrm{g} / \mathrm{mL})$ & \\
\hline 60551 & $18.0 \pm 0.6$ & $5.0 \pm 0.0^{*}$ & 72.2 \\
\hline 66785 & $21.0 \pm 5.2$ & $4.7 \pm 0.6^{\star}$ & 77.6 \\
\hline 75227 & $9.7 \pm 0.6$ & $4.3 \pm 0.6^{\star}$ & 55.7 \\
\hline 97885 & $10.0 \pm 1.0$ & $5.0 \pm 0.0^{\star}$ & 50.0 \\
\hline 82468 & $23.3 \pm 1.2$ & $6.7 \pm 0.6^{\star}$ & 71.2 \\
\hline 02470 & $22.3 \pm 3.8$ & $7.3 \pm 0.6^{\star}$ & 67.3 \\
\hline 09707 & $23.0 \pm 5.3$ & $5.0 \pm 0.0^{*}$ & 78.3 \\
\hline 56053 & $21.3 \pm 6.7$ & $5.3 \pm 0.6^{\star}$ & 75.1 \\
\hline 31829 & $14.0 \pm 1.0$ & $5.0 \pm 1.0^{*}$ & 64.3 \\
\hline 77660 & $23.3 \pm 6.7$ & $4.7 \pm 0.6^{\star}$ & 79.8 \\
\hline 80466 & $14.0 \pm 1.7$ & $5.0 \pm 0.0^{\star}$ & 64.3 \\
\hline
\end{tabular}

Values are mean of two independent experiments. Asterisks indicate significant values compared with control ( $p \leq 0.05)$. CTR, control (L. monocytogenes strains grown in the absence of EO). inoculum of viable bacteria. Experiments were carried out in triplicate.

\section{Cytopathogenic Effects on Caco-2 Monolayers}

The ability of listeriae to cause cytopathogenic effects (CPE) in Caco-2 monolayers was evaluated by the trypan blue exclusion assay (Gibco), which measures cell viability (Facinelli et al., 1998). Briefly, Caco-2 monolayers grown in slide flasks (Corning Costar) were infected with bacterial strains grown with and without the C. sativa EO, as described above. Control monolayers received MEM without bacteria. At the end of the infection period $(1 \mathrm{~h})$, the supernatant was removed and monolayers were washed and covered with MEM supplemented with gentamicin $(10 \mu \mathrm{g} / \mathrm{mL})$. After overnight incubation at $37^{\circ} \mathrm{C}$ in $5 \% \mathrm{CO}_{2}, 1 \mathrm{~mL}$ of a $0.4 \%$ trypan blue solution was added to the culture medium and cells were kept at room temperature for $30 \mathrm{~min}$. Finally, the culture medium with the dye was removed and monolayers were examined with a Leica DMRB microscope (Leica Microsystems, Wetzlar, Germany) using a $10 \times$ objective.

\section{Galleria mellonella Survival Assays}

Final instar larvae $(\sim 300 \mathrm{mg})$ of the greater wax moth $G$. mellonella were purchased from a local vendor, stored in the dark at $15^{\circ} \mathrm{C}$, and used within 7 days. Larvae were rinsed with $70 \%$ EtOH before injection using a standard protocol (Mukherjee et al., 2010). Ten larvae were randomly allocated to different treatment groups: positive control (larvae inoculated with $L$. monocytogenes); test group (larvae injected with $L$. monocytogenes grown in presence of the $C$. sativa $\mathrm{EO}$ ); negative control (larvae that were not injected); PBS control (larvae injected with PBS). Before infection, bacteria were washed and resuspended in PBS at the appropriate cell density $\left(5 \times 10^{7}\right.$ $\mathrm{CFU} / \mathrm{mL})$; then, $50 \mu \mathrm{L}$ of the inoculum was injected into the larval haemocoel via the left proleg using a microsyringe (Mukherjee et al., 2010). After injection, larvae were incubated at $30^{\circ} \mathrm{C}$ in sterile Petri plates. The number of dead larvae was scored at $24 \mathrm{~h}$ intervals for 6 days. Larvae were considered dead when they turned black and failed to react to touch. Three separate experiments were conducted. G. mellonella survival data were plotted using the Kaplan-Meier method.

\section{Statistics}

Data are mean \pm standard deviation (SD). Differences between groups were assessed using GraphPad Prism, version 5 (GraphPad Software Inc., San Diego, CA, USA) by a paired Student's $t$-test (biofilm, cell invasion and real time RT-PCR experiments) and the log-rank (Mantel-Cox) test (G. mellonella survival assays). $P \leq 0.05$ indicated statistical significance.

\section{RESULTS}

\section{GC-MS Analysis of the C. sativa EO}

GC-MS analysis of the C. sativa EO, obtained from the Futura 75 genotype by steam distillation, identified 35 compounds, which accounted for $95 \%$ of the whole GC profile (Table 3). Most were sesquiterpenes found at very low concentrations, except 
for $\beta$-caryophyllene (15.31\%) and $\alpha$-humulene (3.68\%). Myrcene (17.17\%), $\alpha$-pinene (19.20\%), and $\beta$-pinene (4.34\%) were the main monoterpenes.

\section{Susceptibility of $L$. monocytogenes Strains to the C. sativa EO and Its Main Components}

The antimicrobial activity (MIC and MBC) of the total EO, $\alpha$-pinene, and myrcene was determined against all tested strains. Only the MBC values are reported, because the MIC values were difficult to interpret due to turbidity (Table 1 ). The MBC of the whole $\mathrm{EO}$ and of myrcene were $\geq$ $2048 \mu \mathrm{g} / \mathrm{mL}$, whereas those of $\alpha$-pinene ranged from 1024 to $>$ $2048 \mu \mathrm{g} / \mathrm{mL}$, suggesting that both components contribute to the bactericidal activity of the extract against the L. monocytogenes strains.

\section{Reduced Flagellar Motility in Presence of the C. sativa EO}

L. monocytogenes motility was first evaluated by measuring colony diameter and by the "umbrella motility test" after growth on soft agar containing a sublethal concentration $(256 \mu \mathrm{g} / \mathrm{mL})$ of the C. sativa EO (Figures 1A,B). A significant, concentration-dependent, reduction in colony diameter (from 50.0 to $79.8 \%$ ) was observed in all strains (Table 4, Figure 1A). Moreover, strains grown in presence of $256 \mu \mathrm{g} / \mathrm{mL}$ of the extract did not show the typical umbrella-like growth
(Figure 1B). LM examination after flagella staining showed that, unlike control strains, the listeriae incubated with $256 \mu \mathrm{g} / \mathrm{mL}$ of the C. sativa EO formed aggregates with the flagella trapped inside the cluster (Figure 2A). SEM observation confirmed these findings and demonstrated that there were fewer flagella in the extract-incubated strains (Figure 2B).

\section{Downregulation of $\operatorname{prf} A, f l a A, \operatorname{mot} A$, and motB in $L$. monocytogenes Exposed to the C. sativa EO}

Investigation of the relative expression levels of $\operatorname{prf} A, f l a A, m o t A$ and $\operatorname{mot} B$ genes, after growth in the presence of $C$. sativa $\mathrm{EO}$ at $256 \mu \mathrm{g} / \mathrm{mL}$, by a real-time RT-PCR assay, demonstrated a $3.3,8.1$, and 16.8 reduction in the expression levels of $\operatorname{prfA}$, mot $A$ and $m o t B$, respectively, $(p=0.0541, p=0.0819$, and $p=0.0569$, respectively). Interestingly, the relative expression of flaA also demonstrated a significant 241.5 reduction $(p=0.0002)$ (Figure 3).

\section{Effects of the $C$. sativa EO on $L$. monocytogenes Biofilm Formation}

All $11 \mathrm{~L}$. monocytogenes strains were found to be biofilm producers: 4 strains (\#66785, \#02470, \#09707, and \#82468) were strong producers, 6 (\#56053, \#31829, \#77660, \#97885, $\# 75227$, and \#80466) were moderate producers, and one (\#60551) was a weak producer. To evaluate the ability
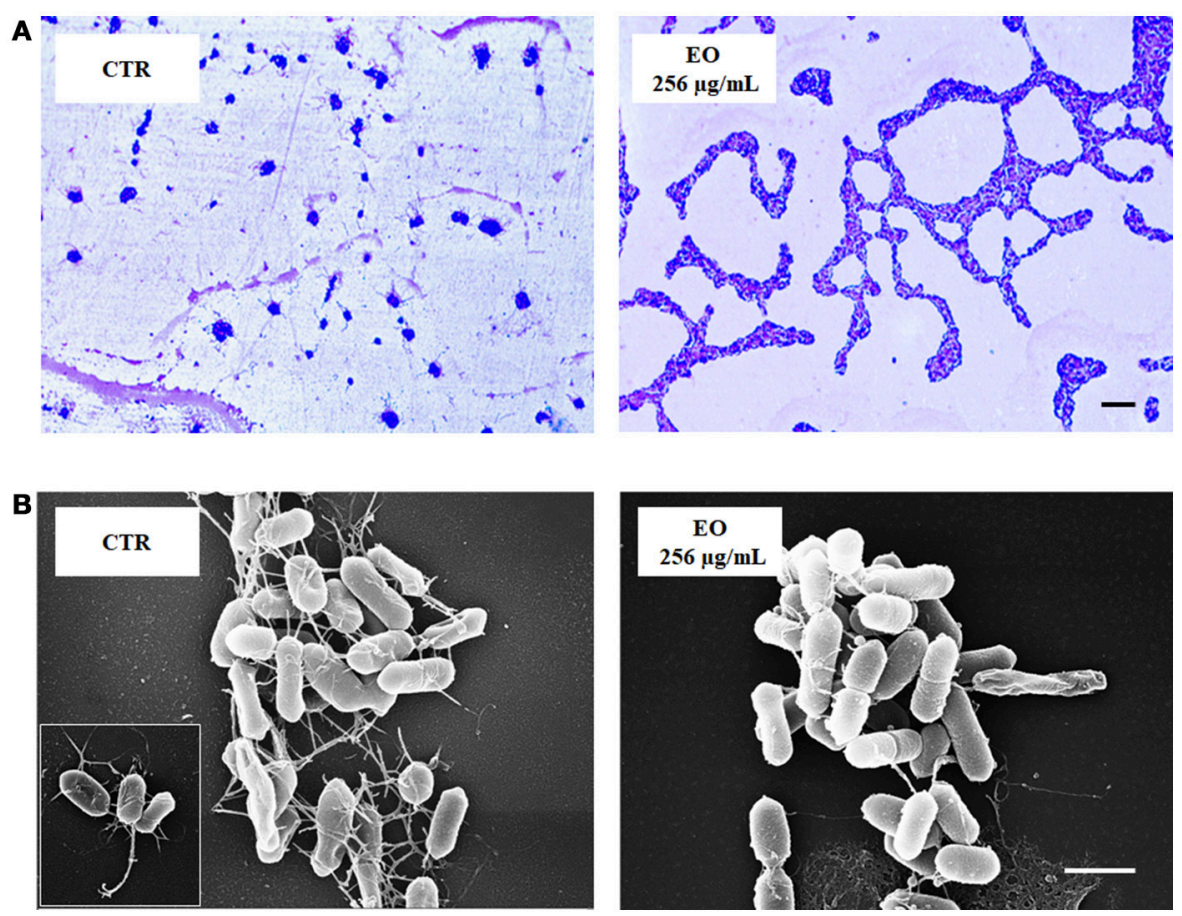

FIGURE 2 | LM and SEM images of L. monocytogenes strain \#80466 grown in presence of the C. sativa EO. (A) LM images after staining with Flagella Stain Droppers (magnification: 1000 ×; bar, $5 \mu \mathrm{m}$ ): flagella are stained in pink red; (B) SEM images (bar, $1 \mu \mathrm{m})$. Insert, "bouquet" of listeriae. CTR, control (L. monocytogenes strain grown in the absence of EO). 
of the EO to interfere with biofilm formation, all strains were tested for biofilm production in presence of sublethal (256, 128, and $64 \mu \mathrm{g} / \mathrm{mL})$ EO concentrations. A reduction in biofilm production was induced by all concentrations in all strains. The $256 \mu \mathrm{g} / \mathrm{mL}$ concentration reduced biofilm production by $25-69 \%$; a similar reduction was observed with $128 \mu \mathrm{g} / \mathrm{mL}$ (from 32 to $73 \%$ ) and $64 \mu \mathrm{g} / \mathrm{mL}$ (from 15 to $65 \%$ ) (Figure 4). Except \#60551 (weak producer), all the moderate/strong biofilm producers became weak producers at all EO concentrations.

A

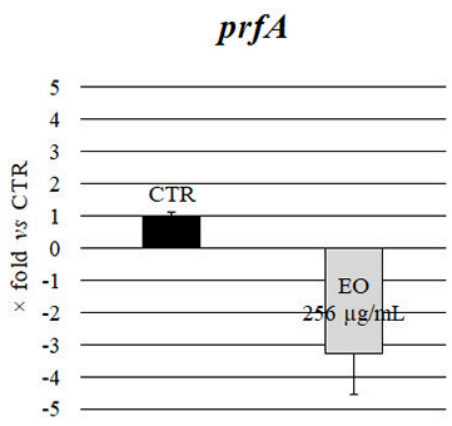

B

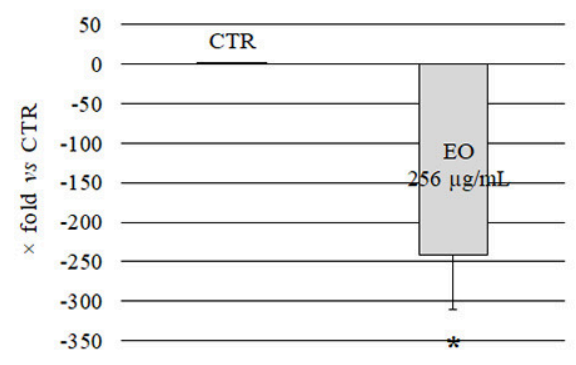

C

$\operatorname{mot} A B$

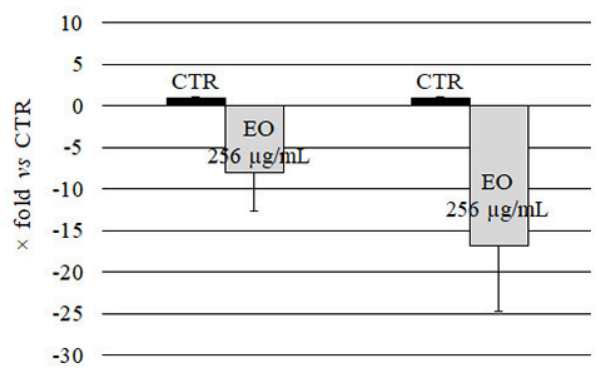

FIGURE 3 | Expression of motility genes of L. monocytogenes strain \#80466 grown in presence of the C. sativa EO. Gene expression was normalized to the expression of the $16 \mathrm{~S}$ rRNA gene in the same conditions compared to the control and is presented as $\mathrm{x}$-fold: (A) prfA; (B) flaA; (C) $m o t A$ and $m o t B$. Mean values and standard deviations of three experiments. Asterisks indicate significant values compared with control $(p \leq 0.05)$. CTR, control (L. monocytogenes strain grown in the absence of EO).

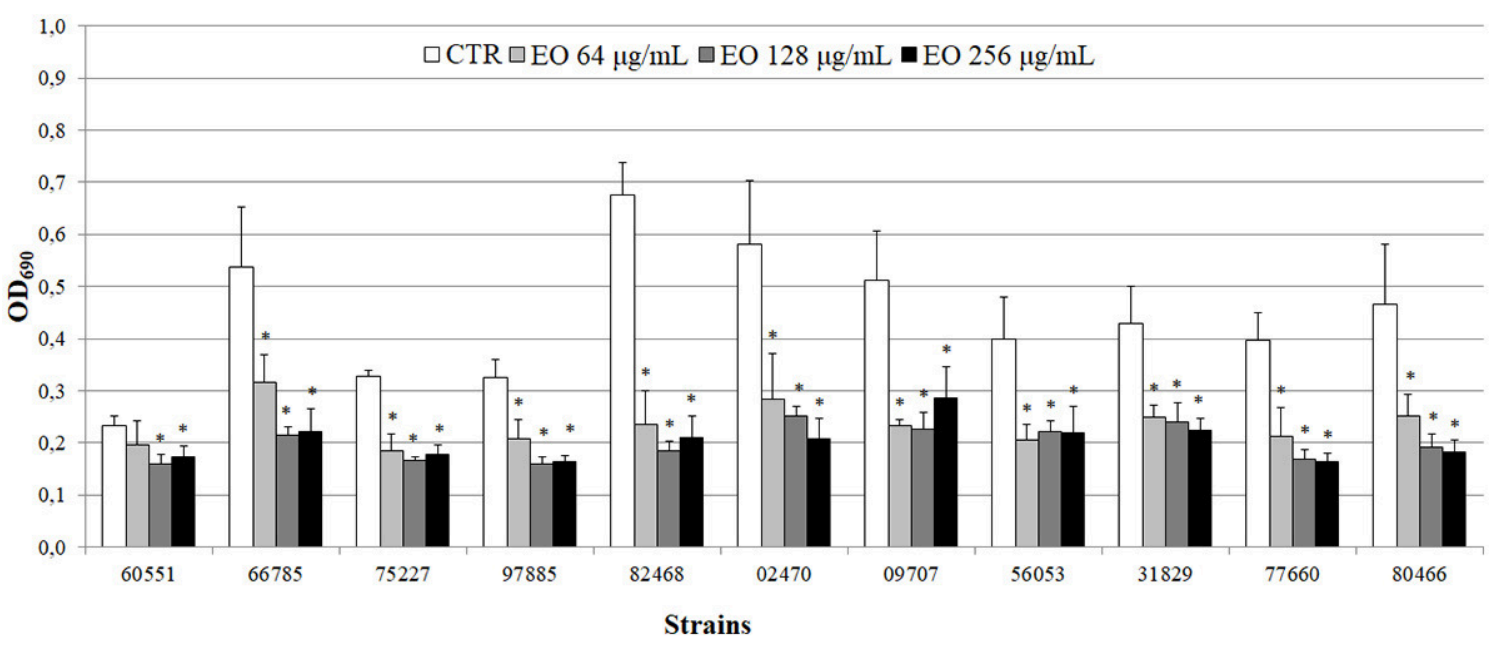

FIGURE 4 | Biofilm production by L. monocytogenes in presence of the $C$. sativa EO. Exposure to the three EO concentrations resulted in a shift to a lower producer type in all but one (\#60551) of the 11 strains. Values are mean $\mathrm{OD}_{690} \pm \mathrm{SD}$ of three experiments. Asterisks indicate significant values ( $\left.p \leq 0.05\right)$ compared with control (L. monocytogenes strain grown in the absence of EO). 


\section{Effects of Exposure of $L$. monocytogenes Strains to the C. sativa EO on Caco-2 Cell Invasion Efficiency and CPE}

Preliminary Caco-2 infection experiments were conducted to ascertain the ability of L. monocytogenes strains to enter Caco-2 cells in the gentamicin survival test. Three strains were excluded from further experiments due to low invasion efficiency; the other 8 strains, which showed an invasion efficiency up to $4.1 \%$ of the initial inoculum, were used in infection inhibition experiments. Remarkably, the invasion efficiency of Caco-2 cells by $7 / 8$ strains was strongly reduced (from 25.9 to $97 \%$ ) when bacteria were grown in presence of $256 \mu \mathrm{g} / \mathrm{mL}$ of the C. sativa EO (Figure 5).

The viability of infected Caco-2 cells was assessed with trypan blue, which stains dead cells but is actively excluded from viable eukaryotic cells. The test demonstrated a dramatic $\mathrm{CPE}$ reduction in L. monocytogenes strains grown in presence of $256 \mu \mathrm{g} / \mathrm{mL}$ of the C. sativa EO (Figure 6), in particular, the monolayers infected with the extract-exposed strains were actually indistinguishable from the uninfected monolayers, whereas the CPE of control monolayers, i.e., those infected with strains grown without the EO, took the form of large, almost contiguous, stained areas.

\section{Survival of G. mellonella Larvae Infected With $L$. monocytogenes Grown in Presence of the C. sativa EO}

The ability of a sublethal concentration of the C. sativa $\mathrm{EO}$ to attenuate $L$. monocytogenes virulence was tested in vivo using the G. mellonella model. Larvae were infected with L. monocytogenes strains grown with and without (control) $256 \mu \mathrm{g} / \mathrm{mL}$ of the C. sativa EO. Larvae were considered dead when they turned black and did not respond to touch. On day 6 after infection with listeriae, larval mortality was about $50 \%$ in the group infected with the strains grown without the EO whereas, remarkably, survival rates greater than $90 \%(p=0.0009)$ were seen in the group infected with the strains grown with the EO (Figures 7A,B). PBS (negative control) did not result in larval mortality (data not shown).

\section{DISCUSSION}

C. sativa L. has been a source of food, fuel, paper, and building materials, a textile fiber, and a folk medicine remedy for thousands of years, and was eventually abandoned due to its high content in psychotropic compounds. Currently, industrial varieties containing low $\triangle 9$-THC concentrations are being studied for their powerful bioactive phytochemicals (Andre et al., 2016).

The antibacterial activity of freshly extracted EO from industrial C. sativa varieties has been assessed by Nissen et al. (2010) against Gram-positive and Gram-negative bacteria, mostly food-borne pathogens. In this study, GC-MS analysis of the EO obtained by distillation of the inflorescences and leaves of the variety Futura 75 disclosed a chemical composition similar to the one reported by Nissen et al. (2010) for this variety. Susceptibility experiments, carried out by the microdilution method, demonstrated that the EO and its two main components, $\alpha$-pinene and myrcene, showed moderate bactericidal activity against clinical strains of $L$. monocytogenes.

The study also evaluated the effects of sublethal concentrations of the C. sativa $\mathrm{EO}$ on L. monocytogenes virulence traits such as motility, biofilm production, and cell

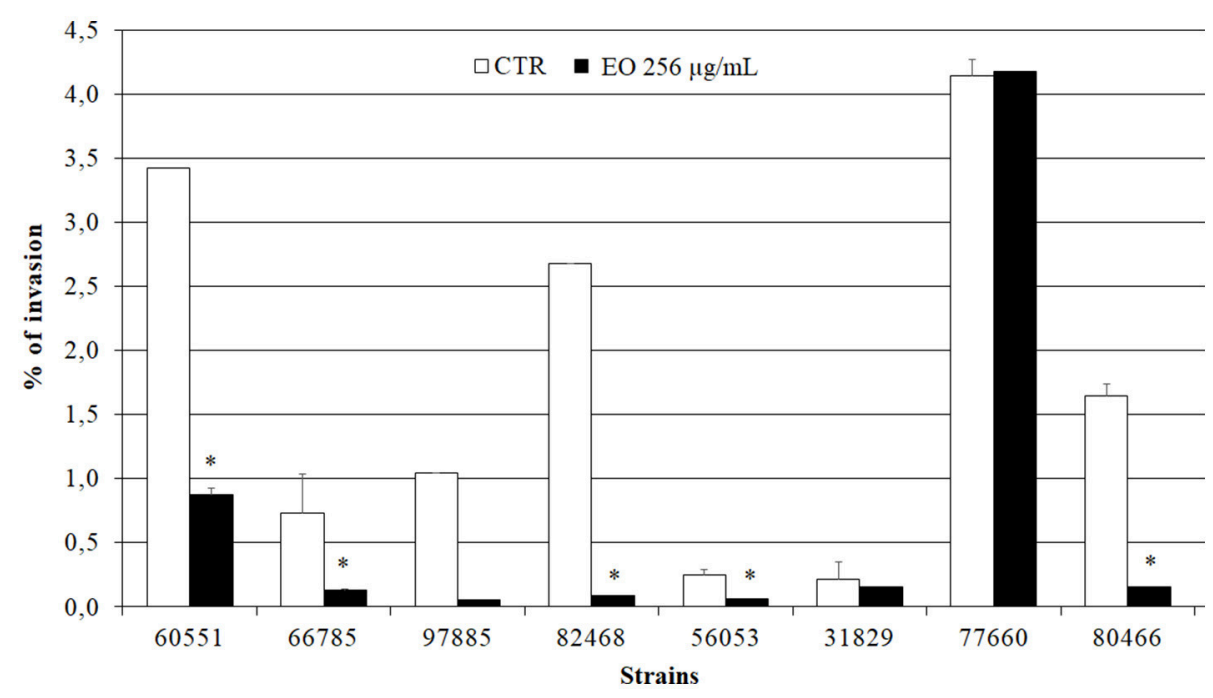

FIGURE 5 | Caco-2 cell invasion by L. monocytogenes strains grown in presence of the C. sativa EO. Data are expressed as percentage (compared with the initial inoculum) of viable bacteria recovered after $2 \mathrm{~h}$ incubation with gentamicin (gentamicin survival test). Each column is the mean of three experiments. Asterisks indicate significant values $(p \leq 0.05)$ compared with control (L. monocytogenes strains grown in the absence of EO). 

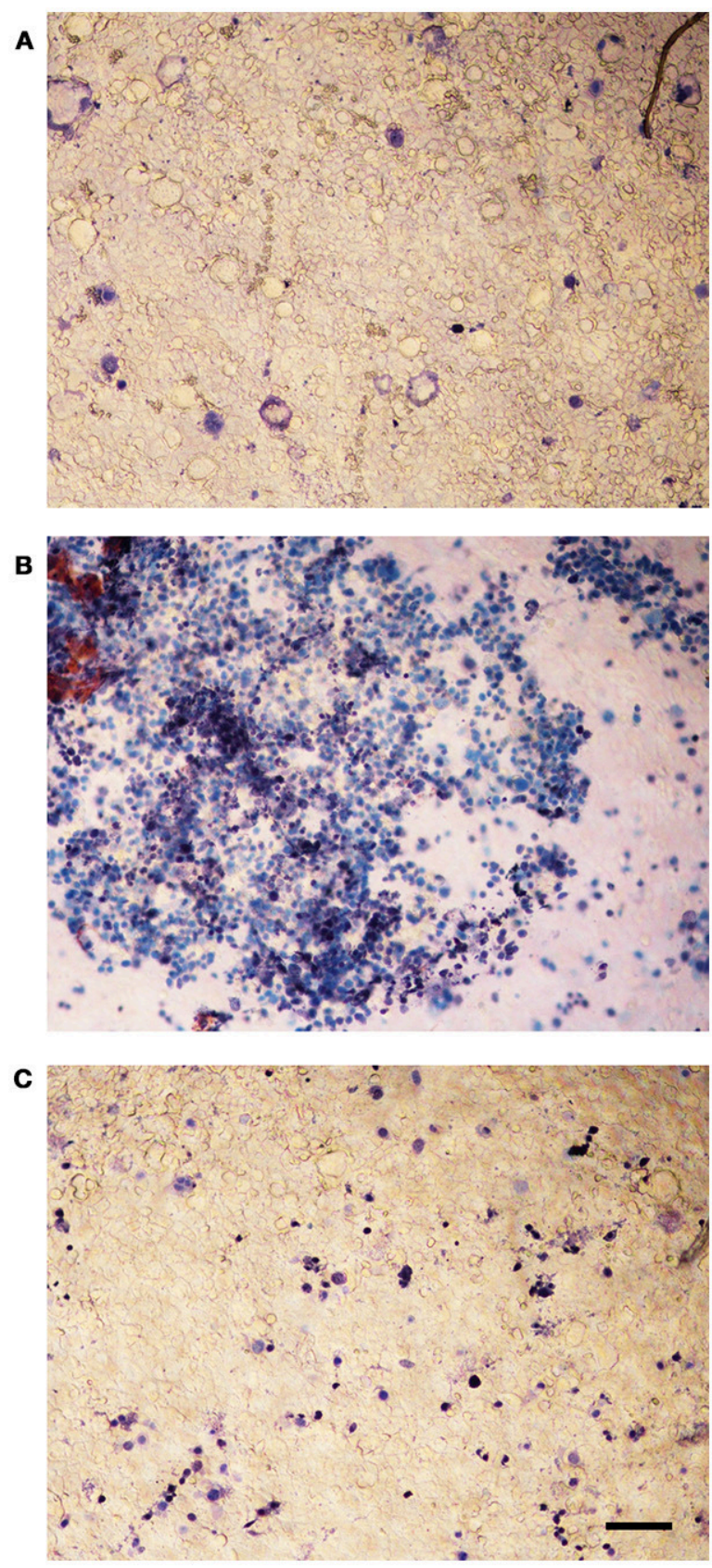

FIGURE 6 | Trypan blue assay on Caco-2 monolayers infected with L. monocytogenes grown in presence of the C. sativa EO. (A) uninfected control; (B) monolayers infected with L. monocytogenes \#80466 not incubated with the EO; (C) monolayers infected with L. monocytogenes \#80466 grown in presence of $256 \mu \mathrm{g} / \mathrm{mL}$ of the EO (magnification: $100 \times$; bar, $400 \mu \mathrm{m}$ ).

invasion. Motility was assessed by phenotypic (umbrella and soft agar motility assay) and molecular (real time RT-PCR) approaches and by LM and SEM observation. Both motility tests demonstrated that, after growth in presence of the $C$. sativa EO, all listerial strains became non-motile. Remarkably, flagella staining of treated cells showed aggregates of listeriae with the flagella trapped inside the aggregates; SEM demonstrated that the flagella were adherent to the cell rather than free, as in control specimens. Real time RT-PCR experiments showed a downregulation of flagellar motility genes flaA (encoding flagellin) and $\operatorname{mot} A$ and $\operatorname{mot} B$ (encoding a part of the flagellar motor). A reduced expression levels of $\operatorname{prf} A$, the transcriptional activator of genes involved in cell invasion, intracellular surviving, and spreading to neighboring cells, was also demonstrated after growth in the presence of $C$. sativa EO. Inhibition of L. monocytogenes motility by natural products has also been reported with trans-cinnamaldehyde from Cinnamomum zeylandicum, carvacrol, and thymol, the main components of oregano and thyme EO (Upadhyay et al., 2012). In the Gram-negative pathogens Salmonella Typhimurium and Escherichia coli O157:H7, carvacrol affects motility through loss of flagellum functionality (Inamuco et al., 2012) and inhibition of flagellin synthesis (Burt et al., 2007), respectively.

Since flagella and flagellum-mediated motility are important virulence traits of L. monocytogenes for initial surface attachment and subsequent biofilm formation (Lemon et al., 2007) as well as for cell invasion (Dons et al., 2004), we tested the hypothesis that a reduction of motility by sublethal concentrations of the $C$. sativa EO would affect biofilm-forming and cell invasion ability. The hypothesis was successfully demonstrated. Remarkably, the number of intracellular listeriae (gentamicin survival test) was almost zero when Caco-2 cells were infected with listeriae exposed to the EO and their viability was unaffected (trypan blue test). The present results therefore clearly demonstrate that sublethal concentrations of the $C$. sativa EO can affect the virulence of $L$. monocytogenes in vitro.

In vivo experiments using $G$. mellonella larvae as a model (Rakic Martinez et al., 2017) were performed to establish whether incubation of listeriae with sublethal concentrations of the $C$. sativa EO would prevent larval infection. The test has recently emerged as a promising model to assess the virulence of numerous human pathogens, including L. monocytogenes. In our study, larvae infected with L. monocytogenes grown in presence of sublethal EO concentrations showed a significantly higher survival compared with larvae infected with untreated listeriae. Thus, the in vivo experiments confirmed the protective activity of the $C$. sativa $\mathrm{EO}$ against $L$. monocytogenes infection.

Since LLO production is critical to L. monocytogenes pathogenesis in G. mellonella (Joyce and Gahan, 2010) we could hypothesize that the protective activity of the C. sativa EO is due to the attenuation of this virulence factor.

Targeting microbial virulence rather than survival is an exciting novel strategy with the potential to reduce the evolutionary pressure for the development of resistance. Food contamination by $L$. monocytogenes remains a major concern for the food processing industry, particularly the plants making ready-to-eat and processed food. The present work provides a baseline in the study of the anti-virulence properties of the EO of $C$. sativa against $L$. monocytogenes. Further studies are needed to understand if it could be used as an alternative agent for the control of L. monocytogenes in food processing plants. 

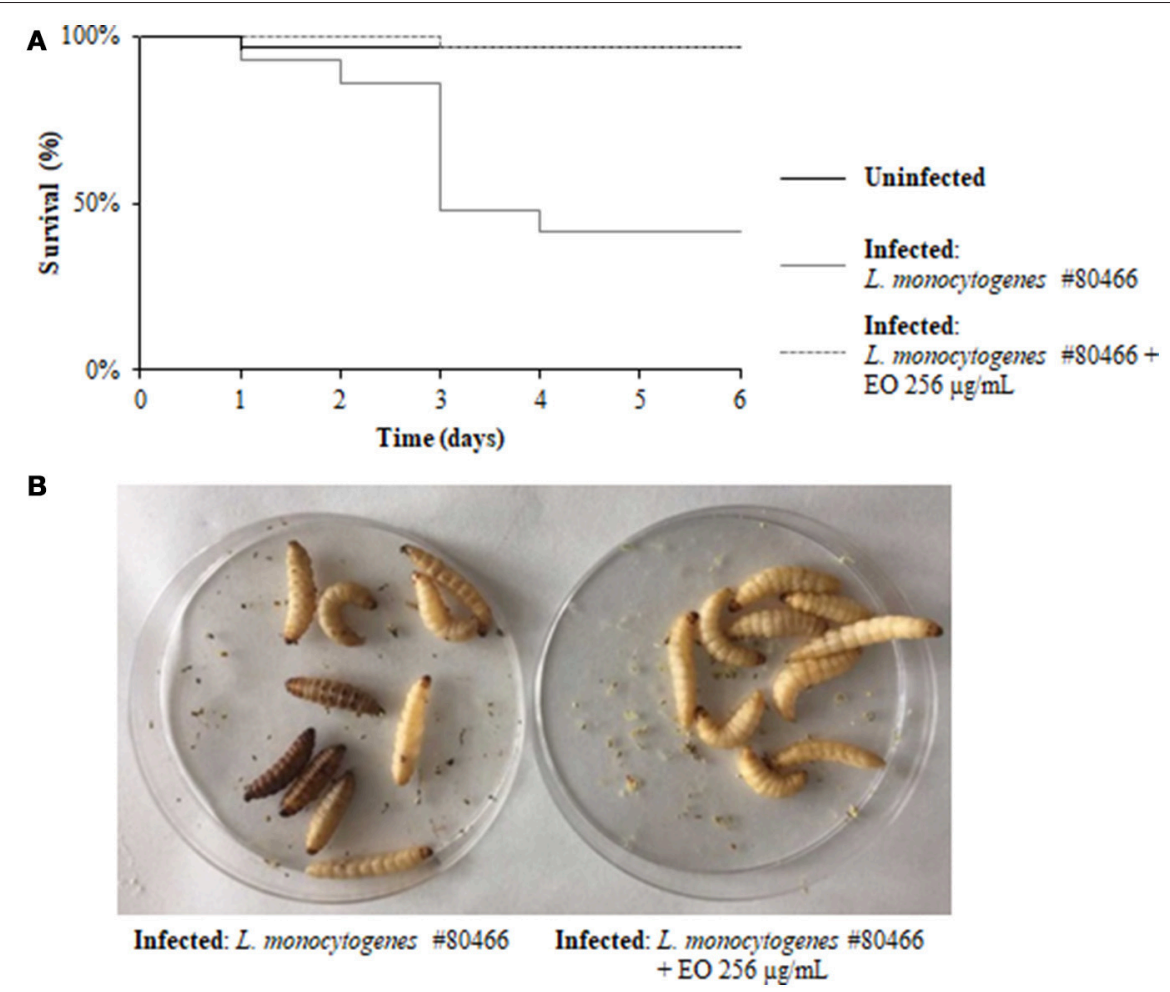

FIGURE 7 | Survival of G. mellonella larvae infected with L. monocytogenes strain \#80466 grown in presence of the C. sativa EO. (A) Kaplan-Meier survival curves of G. mellonella larvae after 6 days from injection with L. monocytogenes strain \#80466 are shown. No more than one control larvae injected with PBS died in any given trial (data not shown); (B) Dead (black and motionless) and viable (yellowish and moving) larvae.

\section{AUTHOR CONTRIBUTIONS}

BF, GM, and EM conceived the study. BF, GM, EM, AP, and MR designed the experiments. GM, EM, AP, and AG performed the experiments. BF, GM, EM, AP, GF, TB, MR, and AG analyzed the data. BF, GM, and EM wrote the paper. All authors approved the manuscript.

\section{FUNDING}

This study was supported by the Project: Plant compounds to fight bacterial antibiotic resistance and virulence:

\section{REFERENCES}

Andre, C. M., Hausman, J. F., and Guerriero, G. (2016). Cannabis sativa: the plant of the thousand and one molecules. Front. Plant Sci. 7:19. doi: 10.3389/fpls.2016.00019

Bierne, H., Milohanic, E., and Kortebi, M. (2018). To be cytosolic or vacuolar: the double life of Listeria monocytogenes. Front. Cell. Infect. Microbiol. 8:136. doi: 10.3389/fcimb.2018.00136

Boqvist, S., Söderqvist, K., and Vågsholm, I. (2018). Food safety challenges and one Health within Europe. Acta Vet. Scand. 60:1. doi: 10.1186/s13028-017-0355-3

Burt, S. A., van der Zee, R., Koets, A. P., de Graaff, A. M., van Knapen, F., Gaastra, W., et al. (2007). Carvacrol induces heat shock protein 60 and inhibits synthesis of flagellin in Escherichia coli O157:H7. Appl. Environ. Microbiol. 73, 4484-4490. doi: 10.1128/AEM.00340-07 searching for bioactive molecules among the agricultural biodiversity of the Marche Region. ERC Field LS6 $43.000,00 €$. Progetto strategico di ricerca di Ateneo 2016 (UNIVPM-DiSBSP) Coordinator: Bruna Facinelli; staff members: Armanda Pugnaloni, Gianna Ferretti, and Tiziana Bacchetti.

\section{ACKNOWLEDGMENTS}

The authors are grateful to Dr. Enrica Cimarelli, President of APPO Marche, for providing the C. sativa L. essential oil.

Cannas, S. (2016). Chemical composition, cytotoxicity, antimicrobial and antifungal activity of several essential oils. Nat. Prod. Res. 30, 332-339. doi: 10.1080/14786419.2015.1060592

Chen, G. Y., Pensinger, D. A., and Sauer, J. D. (2017). Listeria monocytogenes cytosolic metabolism promotes replication, survival, and evasion of innate immunity. Cell. Microbiol. 19:e12762. doi: 10.1111/cmi.12762

Christensen, G. D., Simpson, W. A., Younger, J. J., Baddour, L. M., Barrett, F. F., Melton, D. M., et al. (1985). Adherence of coagulase-negative staphylococci to plastic tissue culture plates: a quantitative model for the adherence of staphylococci to medical devices. J. Clin. Microbiol. 22, 996-1006.

Clinical and Laboratory Standards Institute (2017). Performance Standards for Antimicrobial Susceptibility Testing, 27th Edn. CLSI (Suppl. ) M100. Wayne, PA: USA. 
Colagiorgi, A., Bruini, I., Di Ciccio, P. A., Zanardi, E., Ghidini, S., and Ianieri, A. (2017). Listeria monocytogenes biofilms in the wonderland of food industry. Pathogens 6:41. doi: 10.3390/pathogens60 30041

de las Heras, A., Cain, R. J., Bielecka, M. K., and Vázquez-Boland, J. A. (2011). Regulation of listeria virulence: PrfA master and commander. Curr. Opin. Microbiol. 14, 118-127. doi: 10.1016/j.mib.2011.01.005

Dons, L., Eriksson, E., Jin, Y., Rottenberg, M. E., Kristensson, K., Larsen, C. N., et al. (2004). Role of flagellin and the two-component CheA/CheY system of Listeria monocytogenes in host cell invasion and virulence. Infect. Immun. 72, 3237-3244. doi: 10.1128/IAI.72.6.3237-3244.2004

Drolia, R., Tenguria, S., Durkes, A. C., Turner, J. R., and Bhunia, A. K. (2018). Listeria adhesion protein induces intestinal epithelial barrier dysfunction for bacterial translocation. Cell Host Microbe. 23, 470-484. doi: 10.1016/j.chom.2018.03.004

Duan, Q., Zhou, M., Zhu, L., and Zhu, G. (2013). Flagella and bacterial pathogenicity. J. Basic Microbiol. 53, 1-8. doi: 10.1002/jobm.201100335

EFSA and ECDC (2016). The European Union summary report on trends and sources of zoonoses, zoonotic agents and food-borne outbreaks in 2015. EFSA J. 14:4634. doi: 10.2903/j.efsa.2016.4634

Facinelli, B., Giovanetti, E., Magi, G., Biavasco, F., and Varaldo, P. E. (1998). Lectin reactivity and virulence among strains of Listeria monocytogenes determined in vitro using the enterocyte-like cell line Caco-2. Microbiology 144, 109-118. doi: 10.1099/00221287-144-1-109

Freitag, N. E., Port, G. C., and Miner, M. D. (2009). Listeria monocytogenesfrom saprophyte to intracellular pathogen. Nat. Rev. Microbiol. 7, 623-628. doi: $10.1038 /$ nrmicro2171

Holler, J. M., Bosy, T. Z., Dunkley, C. S., Levine, B., Past, M. R., and Jacobs, A. (2008). $\Delta$ 9-tetrahydrocannabinol content of commercially available hemp products. J. Anal. Toxicol. 32, 428-432. doi: 10.1093/jat/32.6.428

Inamuco, J., Veenendaal, A. K., Burt, S. A., Post, J. A., Tjeerdsma-van Bokhoven, J. L., Haagsman, H. P., et al. (2012). Sub-lethal levels of carvacrol reduce Salmonella Typhimurium motility and invasion of porcine epithelial cells. Vet. Microbiol. 157, 200-207. doi: 10.1016/j.vetmic.2011.12.021

Josenhans, C., and Suerbaum, S. (2002). The role of motility as a virulence factor in bacteria. Int. J. Med. Microbiol. 291, 605-614. doi: 10.1078/1438-4221-00173

Joyce, S. A., and Gahan, C. G. (2010). Molecular pathogenesis of Listeria monocytogenes in the alternative model host Galleria mellonella. Microbiology 156, 3456-3468. doi: 10.1099/mic.0.040782-0

Kathariou, S. (2002). Listeria monocytogenes virulence and pathogenicity, a food safety perspective. J. Food Prot. 65, 1811-1829 doi: 10.4315/0362-028X-65.11.1811

Krawczyk-Balska, A., and Markiewicz, Z. (2016). The intrinsic cephalosporin resistome of Listeria monocytogenes in the context of stress response, gene regulation, pathogenesis and therapeutics. J. Appl. Microbiol. 120, 251-265. doi: $10.1111 /$ jam.12989

Langeveld, W. T., Veldhuizen, E. J., and Burt, S. A. (2014). Synergy between essential oil components and antibiotics: a review. Crit. Rev. Microbiol. 40, 76-94. doi: 10.3109/1040841X.2013.763219

Lemon, K. P., Higgins, D. E., and Kolter, R. (2007). Flagellar motility is critical for Listeria monocytogenes biofilm formation. J. Bacteriol. 189, 4418-4424. doi: 10.1128/JB.01967-06

Lomonaco, S., Nucera, D., and Filipello, V. (2015). The evolution and epidemiology of Listeria monocytogenes in Europe and the United States. Infect. Genet. Evol. 35, 172-183. doi: 10.1016/j.meegid.2015.08.008

Marini, E., Magi, G., Mingoia, M., Pugnaloni, A., and Facinelli, B. (2015). Antimicrobial and anti-virulence activity of capsaicin against erythromycinresistant, cell-invasive Group A Streptococci. Front. Microbiol. 6:1281. doi: $10.3389 /$ fmicb.2015.01281

Marini, E., Magi, G., Vincenzi, C., Manso, E., and Facinelli, B. (2016). Ongoing outbreak of invasive listeriosis due to serotype 1/2a Listeria monocytogenes, Ancona province, Italy, January 2015 to February 2016. Euro Surveill. 21:17. doi: 10.2807/1560-7917.ES.2016.21.17.30217
McDougal, C. E., and Sauer, J. D. (2018). Listeria monocytogenes: the impact of cell death on infection and immunity. Pathogens 7:8. doi: 10.3390/pathogens7010008

Mukherjee, K., Altincicek, B., Hain, T., Domann, E., Vilcinskas, A., and Trinad, C. (2010). Galleria mellonella as a model system for studying Listeria pathogenesis. Appl. Environ. Microbiol. 76, 310-317. doi: 10.1128/AEM.013 01-09

Nazzaro, F., Fratianni, F., De Martino, L., Coppola, R., and De Feo, V. (2013). Effect of essential oils on pathogenic bacteria. Pharmaceuticals 6, 1451-1474. doi: 10.3390/ph6121451

Nissen, L., Zatta, A., Stefanini, I., Grandi, S., Sgorbati, B., Biavati, B., et al. (2010). Characterization and antimicrobial activity of essential oils of industrial hemp varieties (Cannabis sativa L.). Fitoterapia 81, 413-419. doi: 10.1016/j.fitote.2009.11.010

Peel, M., Donachie, W., and Shaw, A. (1988). Temperature-dependent expression of flagella of Listeria monocytogenes studied by electron microscopy, SDS-PAGE and western blotting. J. Gen. Microbiol. 134, 2171-2178.

Radoshevich, L., and Cossart, P. (2018). Listeria monocytogenes: towards a complete picture of its physiology and pathogenesis. Nat. Rev. Microbiol. 16, 32-46. doi: 10.1038/nrmicro.2017.126

Rakic Martinez, M., Wiedmann, M., Ferguson, M., and Datta, A. R. (2017). Assessment of Listeria monocytogenes virulence in the Galleria mellonella insect larvae model. PLoS ONE. 12:e0184557. doi: 10.1371/journal.pone.0184557

Rasko, D. A., and Sperandio, V. (2010). Anti-virulence strategies to combat bacteria-mediated disease. Nat. Rev. Drug Dis. 9, 117-128. doi: $10.1038 / \mathrm{nrd} 3013$

Rousset, M. (1986). The human colon carcinoma cell lines HT-29 and Caco-2: two in vitro models for the study of intestinal differentiation. Biochimie. 68, 1035-1040. doi: 10.1016/S0300-9084(86)80177-8

Silva, L. N., Zimmer, K. R., Macedo, A. J., and Trentin, D. S. (2016). Plant natural products targeting bacterial virulence factors. Chem. Rev. 116, 9162-9236. doi: 10.1021/acs.chemrev.6b00184

Stepanovic, S., Vukovic, D., Dakic, I., Savic, B., and Svabic-Vlahovic, M. (2000). A modified microtiter-plate test for quantification of staphylococcal biofilm formation. J. Microbiol. Methods. 40, 175-179. doi: 10.1016/S0167-7012(00)00122-6

Thønnings, S., Knudsen, J. D., Schønheyder, H. C., Søgaard, M., Arpi, M., Gradel, K. O., et al. (2016). Antibiotic treatment and mortality in patients with Listeria monocytogenes meningitis or bacteraemia. Clin. Microbiol. Infect. 22, 725-730. doi: 10.1016/j.cmi.2016.06.006

Upadhyay, A., Johny, A. K., Amalaradjou, M. A., Ananda Baskaran, S., Kim, K. S., and Venkitanarayanan, K. (2012). Plant-derived antimicrobials reduce Listeria monocytogenes virulence factors in vitro, and down-regulate expression of virulence genes. Int. J. Food Microbiol. 157, 88-94. doi: 10.1016/j.ijfoodmicro.2012.0 4.018

Vázquez-Boland, J. A., Kuhn, M., Berche, P., Chakraborty, T., DomínguezBernal, G., Goebel, W., et al. (2001). Listeria pathogenesis and molecular virulence determinants. Clin. Microbiol. Rev. 14, 584-640. doi: 10.1128/CMR.14.3.584-640.2001

Conflict of Interest Statement: The authors declare that the research was conducted in the absence of any commercial or financial relationships that could be construed as a potential conflict of interest.

Copyright (C) 2018 Marini, Magi, Ferretti, Bacchetti, Giuliani, Pugnaloni, Rippo and Facinelli. This is an open-access article distributed under the terms of the Creative Commons Attribution License (CC BY). The use, distribution or reproduction in other forums is permitted, provided the original author(s) and the copyright owner(s) are credited and that the original publication in this journal is cited, in accordance with accepted academic practice. No use, distribution or reproduction is permitted which does not comply with these terms. 